This item was submitted to Loughborough's Research Repository by the author.

Items in Figshare are protected by copyright, with all rights reserved, unless otherwise indicated.

\title{
Prediction of impulsive vehicle tyre-suspension response to abusive drive- over-kerb manoeuvres
}

PLEASE CITE THE PUBLISHED VERSION

http://dx.doi.org/10.1177/1464419312469756

PUBLISHER

SAGE @ IMechE

VERSION

AM (Accepted Manuscript)

LICENCE

CC BY-NC-ND 4.0

\section{REPOSITORY RECORD}

von Chappuis, Hubertus, George Mavros, P.D. King, and Homer Rahnejat. 2014. "Prediction of Impulsive Vehicle Tyre-suspension Response to Abusive Drive-over-kerb Manoeuvres". figshare.

https://hdl.handle.net/2134/14741. 
This item was submitted to Loughborough's Institutional Repository (https://dspace.lboro.ac.uk/) by the author and is made available under the following Creative Commons Licence conditions.

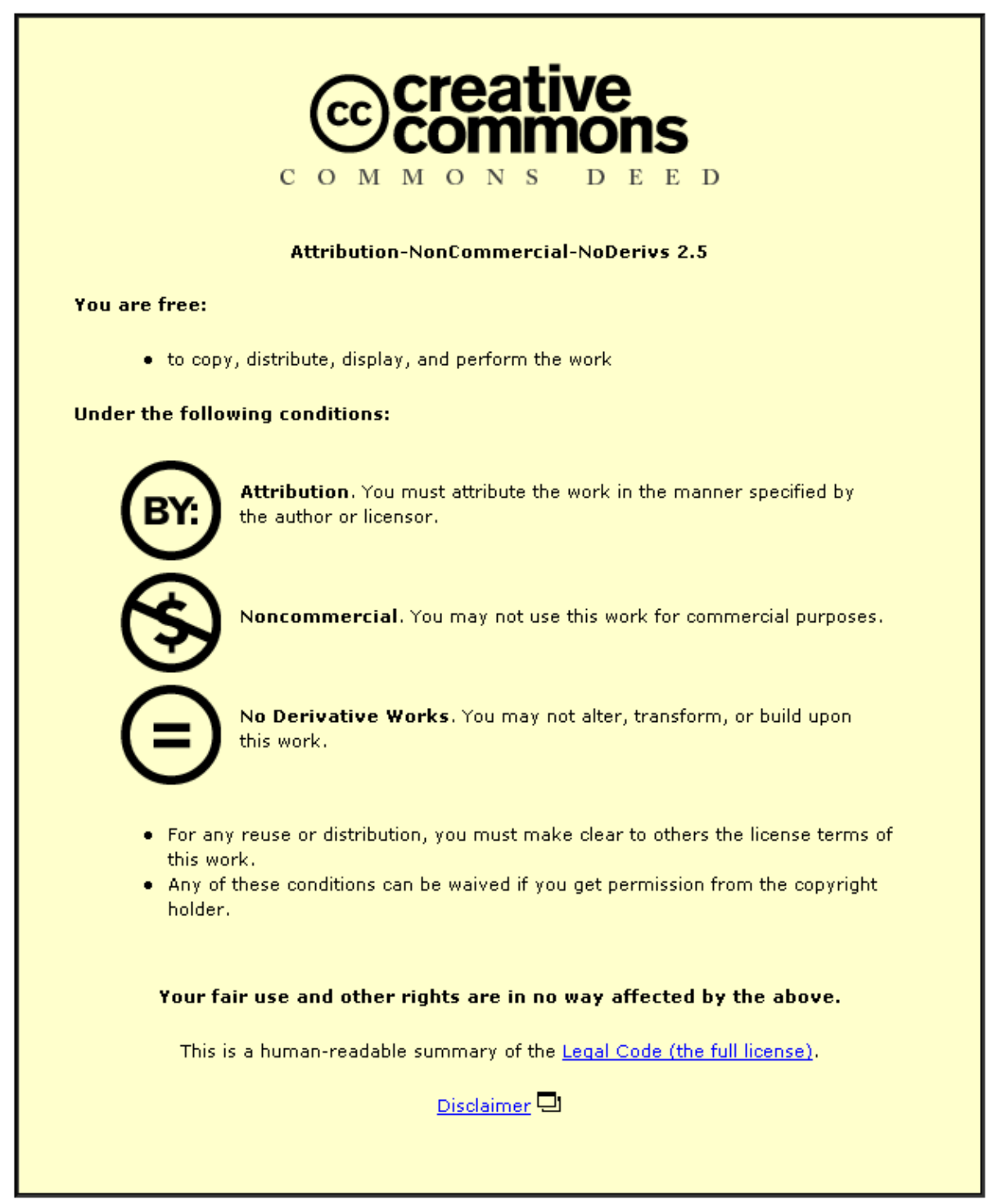

For the full text of this licence, please go to: http://creativecommons.org/licenses/by-nc-nd/2.5/ 


\title{
PREDICTION OF IMPULSIVE TYRE-SUSPENSION RESPONSE SUBJECTED TO KERB STRIKE
}

\author{
Hubertus von Chappuis ${ }^{1}$, George Mavros ${ }^{2}$, Paul King ${ }^{2}$ and Homer Rahnejat ${ }^{2}$ \\ ${ }^{1}$ Ford Werke GmbH, Spessartstraße, D-50725, Cologne-Merkenich, Germany \\ ${ }^{2}$ Loughborough University, Epinal way, Loughborough, LE11 3TU, UK
}

\begin{abstract}
The paper presents a minimal parameter vehicle simulation model to predict the vertical suspension loads expected during abuse driving manoeuvres, such as a kerb strike event. This causes impulsive bump-stop loads. Since the aim is to specifically study impulsive bump-stop and tyre characteristics, an in-plane pitch model with 7 degrees of freedom suffices. Non-linear and hysteretic characteristics of the bump-stop elements are included by a parametric map concept, based on displacement and velocity dependent hysteresis. Furthermore, a static tyre model is described, tailored to predict the radial stiffness against penetration of an edge with a flat-type rigid body geometry. The tyre model is derived on the basis of classical shell theory and represented in terms of only a few input parameters. Model validation is supported through experiments at both component and system levels.
\end{abstract}

Keywords: Minimal parameter vehicle model, abuse manoeuvres, kerb strike 


\section{INTRODUCTION}

With increasing computational power, complex simulation models allow design optimization in the final development phase. However, at an early stage, most details, other than main dimensions and inertias are unknown. For concept studies, for example in the early stages of chassis development, and prediction of component loads, model simplicity is preferred. This approach is followed in the current paper with a minimal parameter vehicle model. In automotive industry the application of such models is of advantage, because of their simple set up, fast run and easy interpretation. The purpose of the model presented here is to estimate an envelope for the expected vertical suspension load level, be it durability [1, 2] or abuse kind [3, 4] applications.

Suspension and tyre characteristics should possess many attributes for ride comfort, durability and vehicle handling. In this paper, emphasis is put upon modelling of jounce bumper characteristics [5, 6] and tyre behaviour $[\mathbf{7 , 8 , 9}$ ] under severe impulsive conditions such as kerb strike (Figure 1), where their elasto-kinetics are usually assessed for various load case scenarios [10]. FORD has specified the worst vertical load case manoeuvre to be drive over kerb (DOC). A 2-dimensional (planar) vehicle model $[\mathbf{1 1}, \mathbf{1 2}]$, extended to take into account fore-aft wheel travel is used. Large scale wheel motions lead to impulsive action which can induce significant jounce bumper impact and gross tyre deflections.

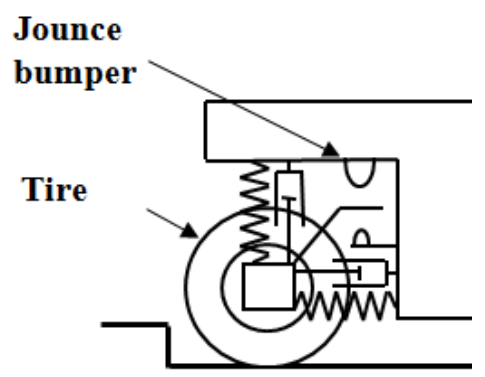

Figure 1: Considered key components for vertical load simulation.

\section{VEHICLE MODEL}

The equations of motion of the model are formulated using a direct Newton-Euler approach. For the sake of simplicity, the vertical and longitudinal suspension compliances are represented by idealized spring and damper elements, no kinematic constraint formulation is required.

\subsection{Model element overview}

The inertias of the suspension links are lumped with the unsprung wheel mass. The effects of suspension geometry, kinematics and stiffness of individual components are merged into combined vertical and longitudinal stiffnesses. Figure 2 shows an overview of the vehicle model. Important features of the model are described in the following list.

(1) Sprung mass and rotational inertia.

(2) Unsprung masses including wheel, hub, brake and lumped suspension.

(3) Road springs (constant stiffness).

(4) Shock absorbers (constant damping).

(5) Jounce bumpers (map concept rolled out in item 4).

(6) Rebound stops with non-linear stiffness (no damping).

(7) Suspension travel constraints in jounce and rebound directions. 
(8) Longitudinal stiffness / damping of wheel centre against body.

(9) Tyre.

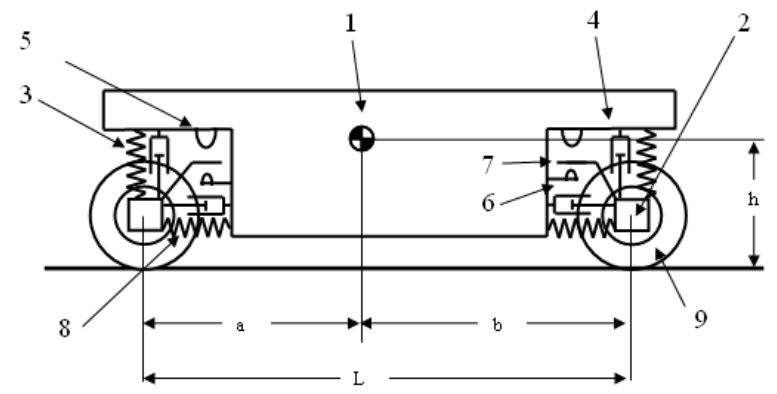

Figure 2: Minimum parameter vehicle model.

\subsection{Equations of motion}

The basis of the model is an in-plane pitch model $[\mathbf{1 1}, \mathbf{1 2}]$, extended by three additional longitudinal degrees of freedom as shown in Figure 3.

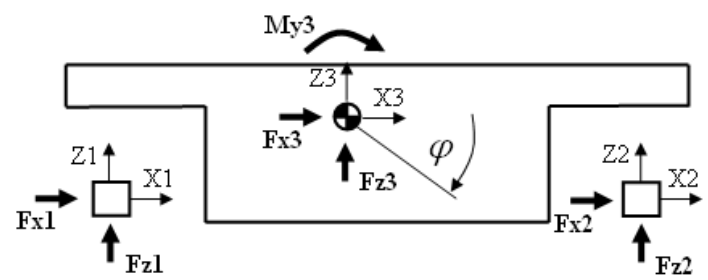

Figure 3: Degrees of freedom and external loads for simple vehicle model.

The equations of motion in a matrix form are:

$[M] \cdot\left\{\begin{array}{c}\ddot{z}_{i} \\ \ddot{x}_{j} \\ \ddot{\varphi}_{k}\end{array}\right\}+[D] \cdot\left\{\begin{array}{c}\dot{z}_{i} \\ \dot{x}_{j} \\ \dot{\varphi}_{k}\end{array}\right\}+[K] \cdot\left\{\begin{array}{c}z_{i} \\ x_{j} \\ \varphi_{k}\end{array}\right\}=\left\{\begin{array}{c}\sum f z_{i} \\ \sum f x_{j} \\ \sum m y_{k}\end{array}\right\}$

$[\mathbf{M}]$ is the inertia matrix, $[\mathbf{K}]$ and $[\mathbf{D}]$ are the effective stiffness and damping matrices. The externally applied forces constitute the RHS of the equation. Matrix [D] is of equivalent structure to [K].

In contrast to typical in-plane models, the system properties of the model shown in Figure 1 vary depending on the level of whel displacement. The full range of wheel displacement is divided into the following three states.

1. free wheel travel (only suspension spring/damper are active)

2. jounce bumper contact

3. rebound stop contact

Figure 4 shows the vehicle front suspension in vertical free travel- (left) and in bump stop engagement mode (right). 


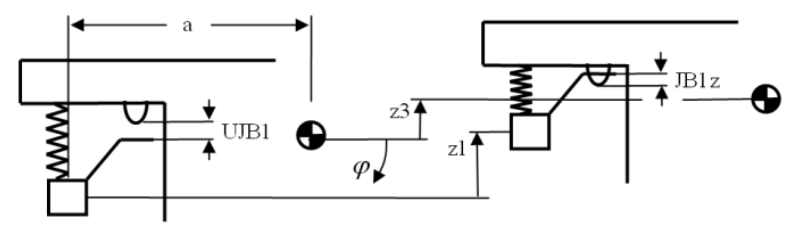

Figure 4: Free vertical wheel travel UJB1 and jounce bumper engagement JB1z on a front suspension.

For the free travel state (case 1 above) the matrices [K] and [D] do not include structural stiffness and damping characteristics associated with jounce and rebound bumpers, which should be included in cases 2 and 3 above. In order to keep the system linear and the stiffness and damping matrices unchanged through the simulation, the effect of jounce bumper or rebound stop engagement is handled by modifying the forces at the right-hand-side of equation (1). Hence, for case 1 (free wheel travel):

$$
\vec{F} e_{(1)}=\left\{\begin{array}{c}
f_{1 z}-m_{1} g \\
f_{2 z}-m_{2} g \\
-m_{3} g \\
f_{1 x} \\
f_{2 x} \\
0 \\
0
\end{array}\right\}
$$

And for cases 2 (jounce bumper engagement) and 3 (rebound stop engagement) at the front suspension:

$$
\vec{F} e_{(2)}=\left\{\begin{array}{c}
f_{1 z}-m_{1} g-F_{J B 1} \\
f_{2 z}-m_{2} g \\
-m_{3} g+F_{J B 1} \\
f_{1 x} \\
f_{2 x} \\
0 \\
F_{J B 1} \cdot a
\end{array}\right\}, \vec{F} e_{(3)}=\left\{\begin{array}{c}
f_{1 z}-m_{1} g+F_{R S 1} \\
f_{2 z}-m_{2} g \\
-m_{3} g-F_{R S 1} \\
f_{1 x} \\
f_{2 x} \\
0 \\
F_{R S 1} \cdot a
\end{array}\right\}
$$

\subsection{Jounce bumper force response map}

The concept of a jounce bumper map is used to feed back the bumper force $F_{J B}$ according to the states $z$ and $\dot{z}=d z / d t$. This map concept takes the non-linearity and hysteresis of these polymeric elements into account and is presented in detail in section 3.

\subsection{External tyre force application}


In kerb strike, the tyre loads are derived from the radial deformation of the tyre's belt. The kerb is assumed to be a simplified vertical rigid blade, promoting an edge-type line contact. Therefore, with this assumption the complexity of a combined flat/edge type contact is removed. Later in section 4 it will be shown that this assumption does not harm the accuracy of tyre force predictions. As done with the jounce bumper and rebound stop forces, tyre forces are included on the right-hand-side term (Fe) of equation (1), as shown in Figure 5.

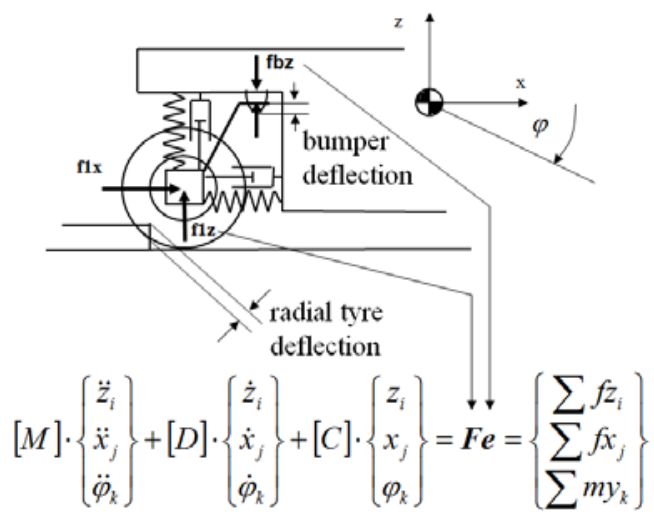

Figure 5: Tyre response force feedback to external load vector Fe.

\section{BUMPER MODEL}

The jounce bumper is regarded as a 1-DOF vertical compression-only element. It attenuates the effect of impact load transmission to the vehicle body post impulsive event, be it kerb or pothole impact. It is made of polyurethane (PUR) foam, commonly used in vehicle suspensions. Its force response characteristic is a combination of both a polytropic deformation of gas chambers and compression of the gas volume surrounding polymeric structure itself. The material properties, such as density depend on the specific boundary conditions applied during the polymerization process. It is, therefore, difficult to investigate the bumper force response by means other than physical testing.

Simple, conventional simulation models consist of a non linear spring element combined with viscous damping [2, 3]. The spring characteristic is based on a quasi static force response trace taken from the bumper element.

Simulations are also performed by using rheological models [5, 6], combining Maxwell-, Kelvin, friction and conventional spring elements to address the hysteretic features of polymer elements. In this paper, an alternative map concept is proposed.

During a kerb strike manoeuvre as described in the introduction, the bumper may deform to its full extent. Although bumper excitation during kerb strike is of the impact type and can vary in terms of exact duration, amplitude and shape of the force trace, a harmonic excitation is assumed in order to obtain the jounce bumper's force response data. The validity of this assumption is confirmed through comparison of the simulation predictions with experimental drop tests, which are considered fairly representative of the excitation during kerb strike.

The quotient $\omega=V \max$ / Smax is used to specify circular actuation frequency relevant for the drive over kerb (DOK) event. With Vmax being limited by the damper blocking speed and Smax specified by the bumper deflection range, $f=\omega / 2 \pi$ determines the DOK relevant frequency to be around $10 \mathrm{~Hz}$. Thus, the recorded data does contain the velocity related damping hysteretic characteristics. Figure 6 shows the bumper actuation kinematics and the physical rig used to generate the map data. 

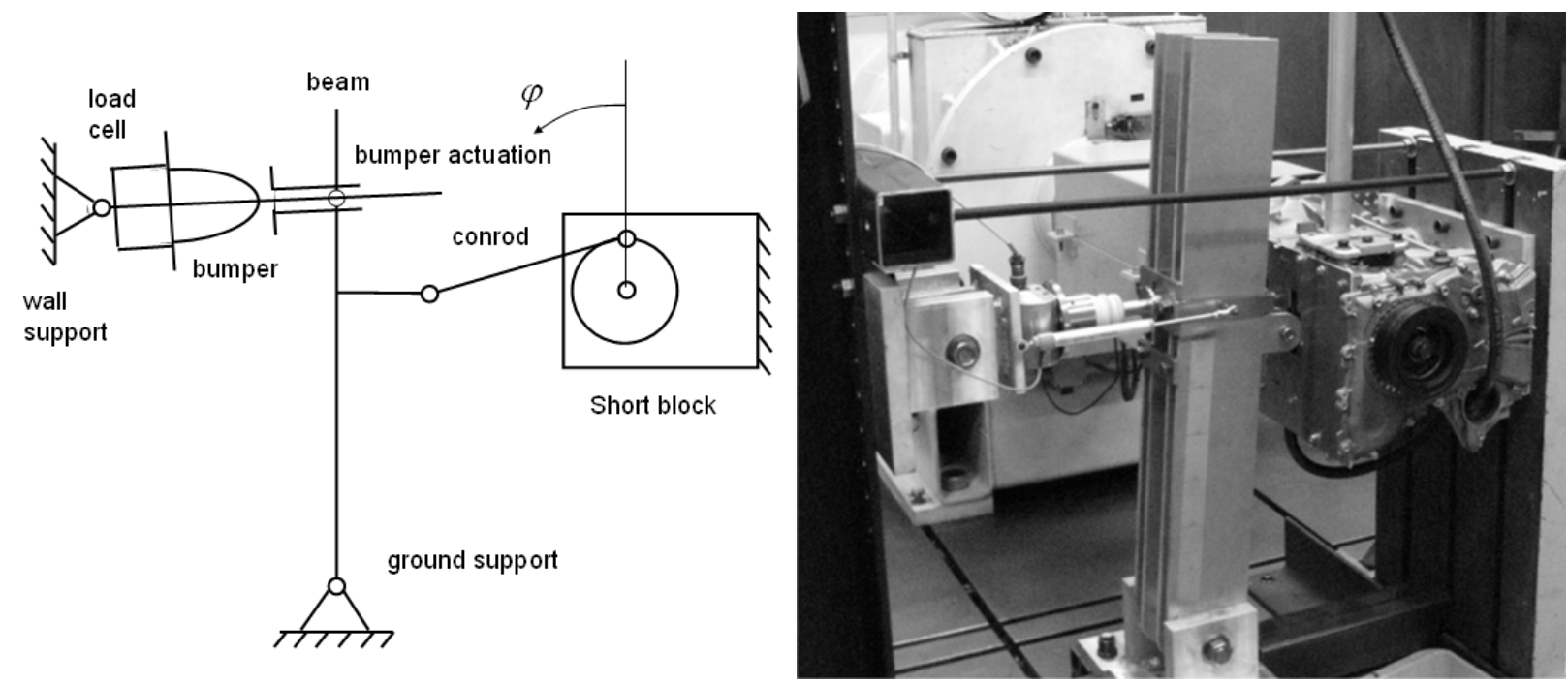

Figure 6: Concept of bumper test rig kinematics and physical test set up.

Any small deviations from harmonic response due to higher order crank kinematics are neglected.

Figure 7 shows the force obtained for various harmonic excitation amplitudes S1 to S7, as a function of the phase (or crank) angle.

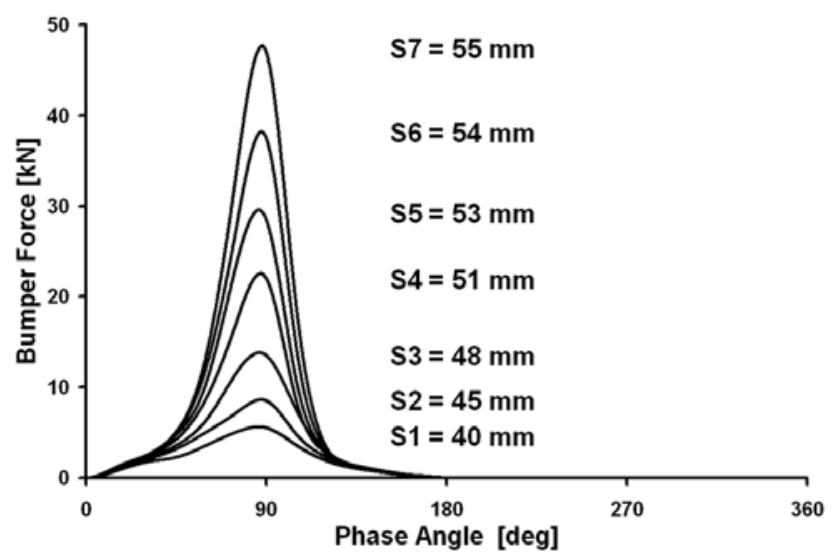

Figure 7: Force response of a jounce bumper element to sinusoidal actuation.

\subsection{Map data pre-processing}

The phase angle as shown in Figure 7 is divided into equal, narrower bands of, say, $10^{\circ}$. For each angle band and for every excitation amplitude S1 to S7, a cubic spline interpolation is used to condense the recorded data into a third order polynomial:

$$
F=A_{0}+A_{1} \cdot z+A_{2} \cdot z^{2}+A_{3} \cdot z^{3}
$$

Variable 'z' represents the crank angle (independent variable) within a narrow band, as illustrated in Figure 8a. The Figure also shows the organisation of data into an easily accessible array, containing the coefficients of the interpolation polynomial (4). 
a)

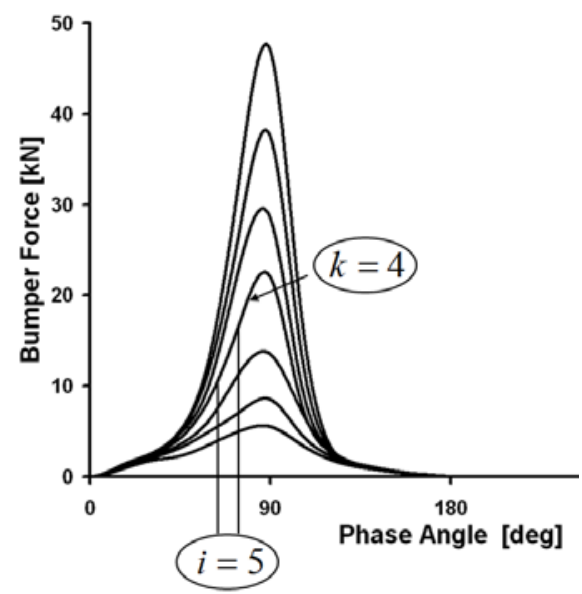

b)

$A 3 D=$

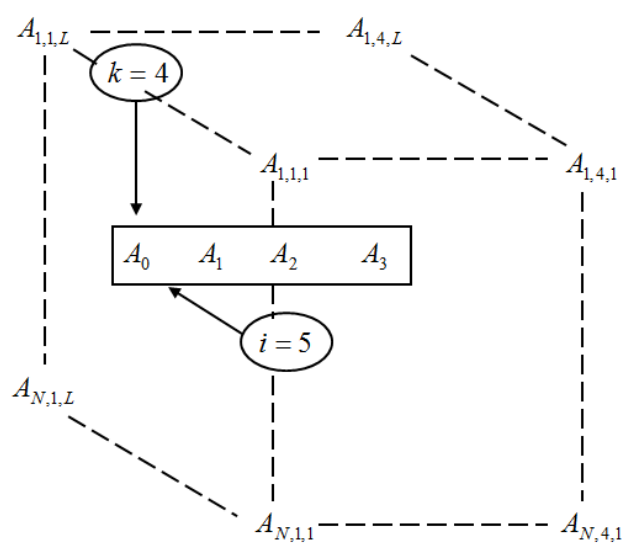

Figure 8: Spline interpolation band (a) and organization of array 'A' to store map data cubical spline coefficients (b).

In the 3D array shown in Figure 8b, each element $A 3 D(i, j, k)$ indicates a specific polynomial coefficient. Index I, indicating downward progression in the array, is associated with the angle band, for example from $70^{\circ}$ to $80^{\circ}$. Index $\mathrm{j}$, indicating progression to the right is associated with the four coefficients of a specific polynomial corresponding to a specific angle band and excitation amplitude. Finally, index k, indicating backward progression in the array, is associated with different excitation amplitudes, S1 to S7.

\subsection{Map-solver interaction}

The stored map feeds back the force response corresponding to the current states vertical suspension deflection and its rate ( $\mathrm{S}$ and $\mathrm{V}$ ) at each integration time step. In conjunction with the expression for the circular frequency phase angle $\varphi=\omega t$, a generalized velocity vg $=v_{(t)} / \omega$ can be defined as:

$$
\begin{aligned}
& S_{(t)}=S_{0} \cdot \sin (\varphi) \\
& \frac{v_{(t)}}{\omega}=S_{0} \cdot \cos (\varphi)
\end{aligned}
$$

The phase diagram of displacement plotted against generalised velocity reveals a format of concentric circles, as illustrated in Figure 9.

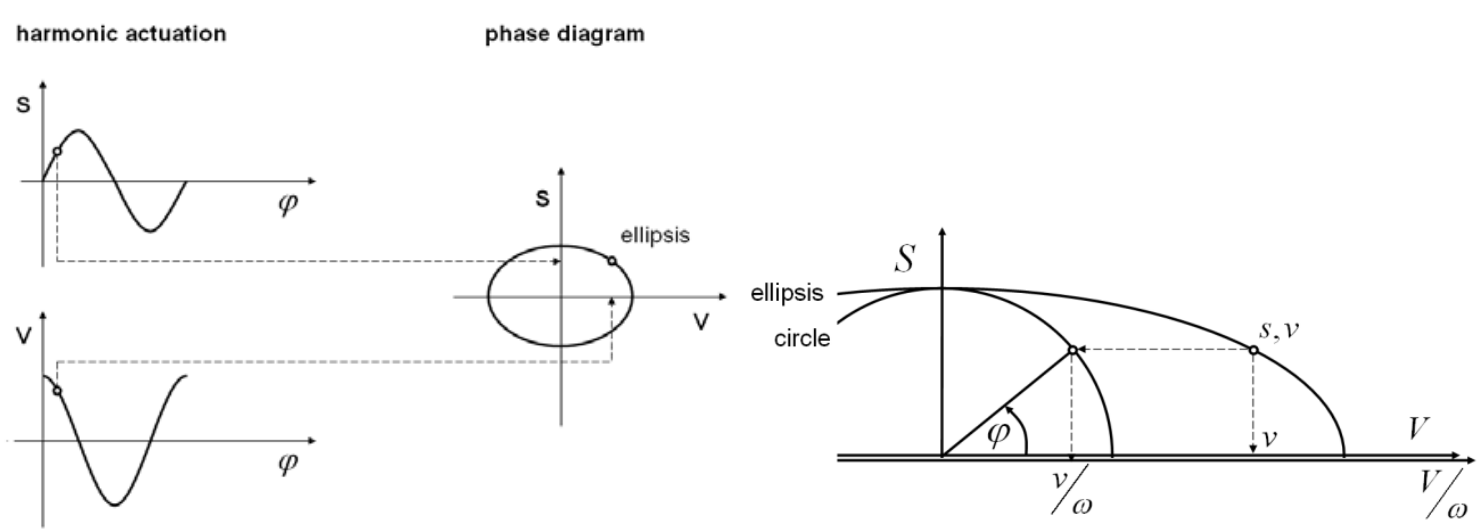

Figure 9: generalized velocity in phase diagram. 
Plotting the force against phase angle for a number of different excitation amplitudes leads to the three dimensional plot shown in Figure 10.

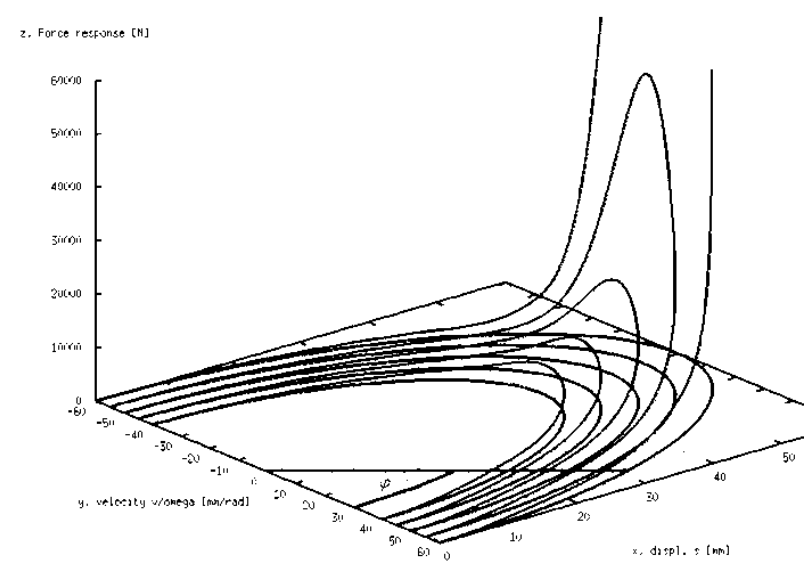

Figure 10: Force response traces $(\mathrm{z})$ and their vertical projections (x, y) on a phase diagram.

Also note that

$\tan \varphi=\frac{S}{V / \omega} \quad R=\sqrt{S^{2}+(V / \omega)^{2}}$

For any pair of states (S, V), $\tan \varphi$ and $\mathrm{R}$ can be obtained by using equations (6) and the angular frequency used for the generation of the map.

The previously defined 3D array, A3D, provides interpolation data allowing the calculation of the force corresponding to any phase angle but discrete amplitudes S1 ... S7 (or radii R). In the event that a pair of states (S, V) falls between concentric circles, a second quadratic interpolation is performed, as shown graphically in Figure 11.

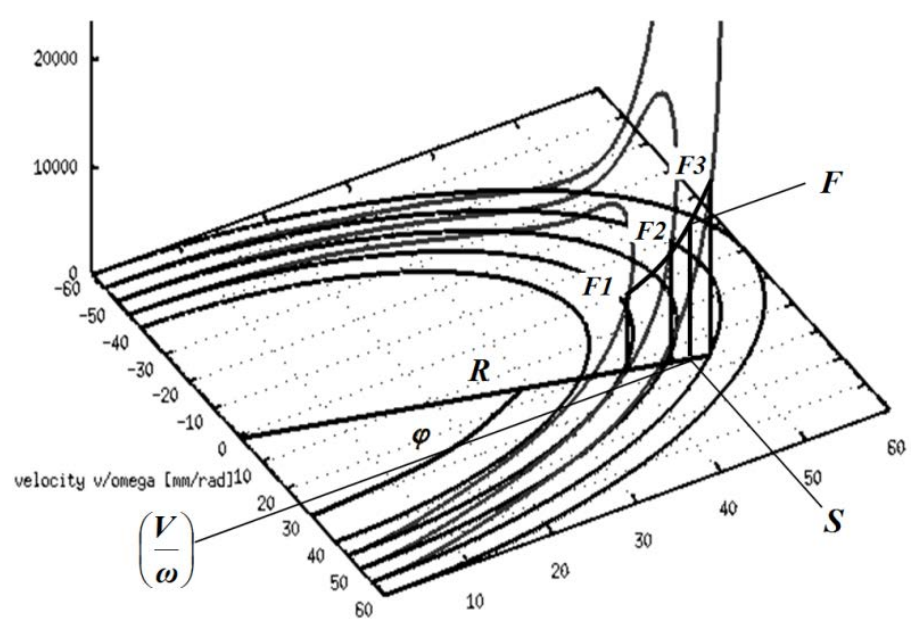

Figure 11: Circumferential and radial interpolation

\subsection{Map concept validation}

To ascertain the validity of the map concept, simulation results are compared with a drop test applied to a bumper element. Figure 12 below shows good agreement between the results obtained by simulation using the harmonically generated map concept and results obtained by physical drop tests. 


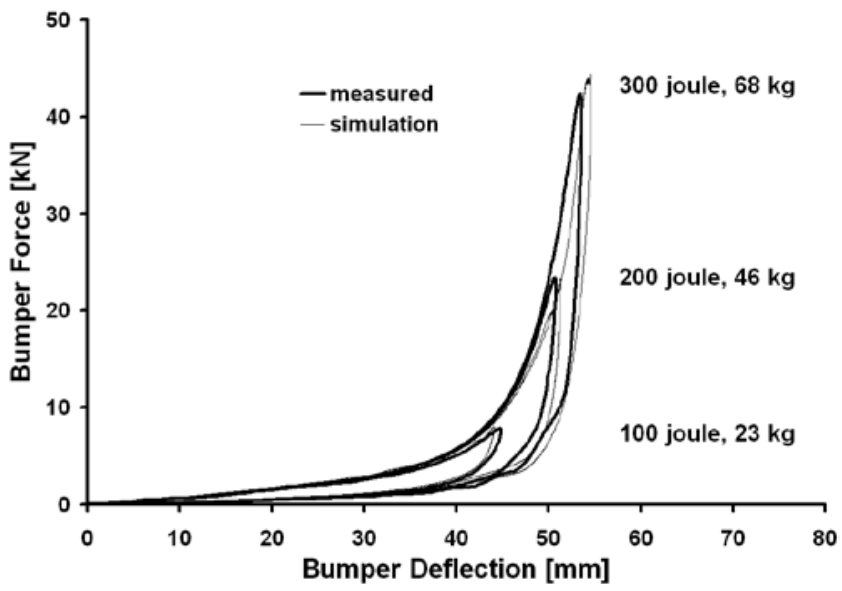

Figure 12: Comparison between force simulation (light) and measured drop test forces (bold trace)

In addition, comparison between the map concept drop and a simple model comprising a non linear spring element in the absence of damping reveals the improvement offered by this method, as illustrated in Figure 13.

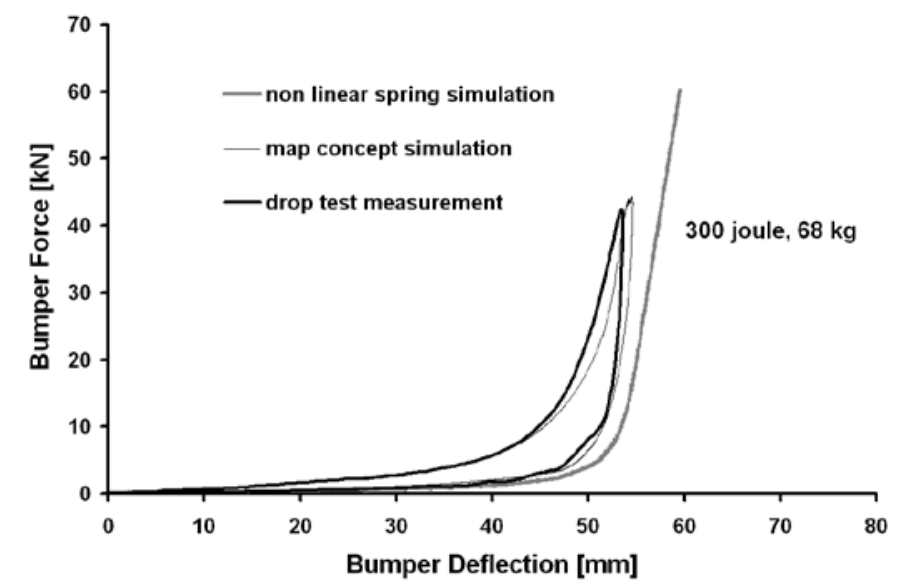

Figure 13: Map concept compared to a simple non linear model.

\section{TYRE MODEL}

The model is simplified to address the radial force response of the tyre, neglecting its structural stiffness and inertia. The tyre structure is simplified by including two primary elements, namely the belt and the sidewall, as shown in Figure 14. The model is fully defined by the pneumatic pressure, p, and four dimensions of the cross section ( $\mathrm{Ra}$, ri, bt and $\mathrm{fc}$ ), as illustrated. The belt and sidewall are considered as ideal isotropic membranes, free from resistance against bending. All tyre model derivations are based on the above simplifications and assumptions. 


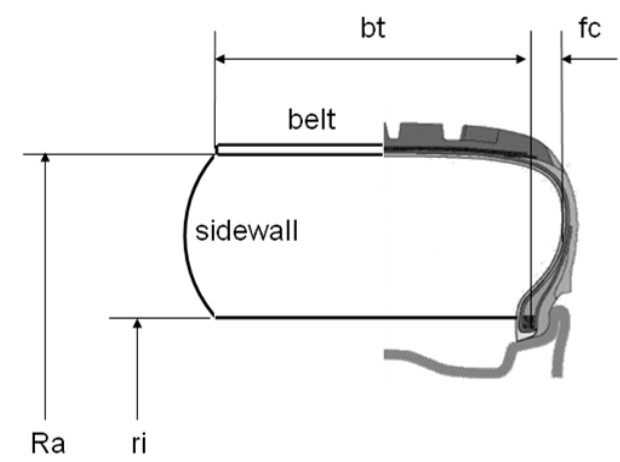

Figure 14: Tyre cross section showing the geometrical parameters required by the model.

\subsection{Pneumatic caused internal stress for an un-deformed tyre}

In the absence of radial deformation, the belt contour is assumed to be of ideal cylindrical shape. The internal radial stress at the circumferential interface between sidewall and belt, as well as the tangential belt force Fg, are derived by making use of shell theory. Timoshenko [13] formulated a general in plane stress relation (7) for a double curved, thin shell element and applied it to a torus structure, as shown in Figure 15.

$$
\frac{\sigma_{1}}{R 1}+\frac{\sigma_{2}}{R 2}=\frac{p}{t}
$$
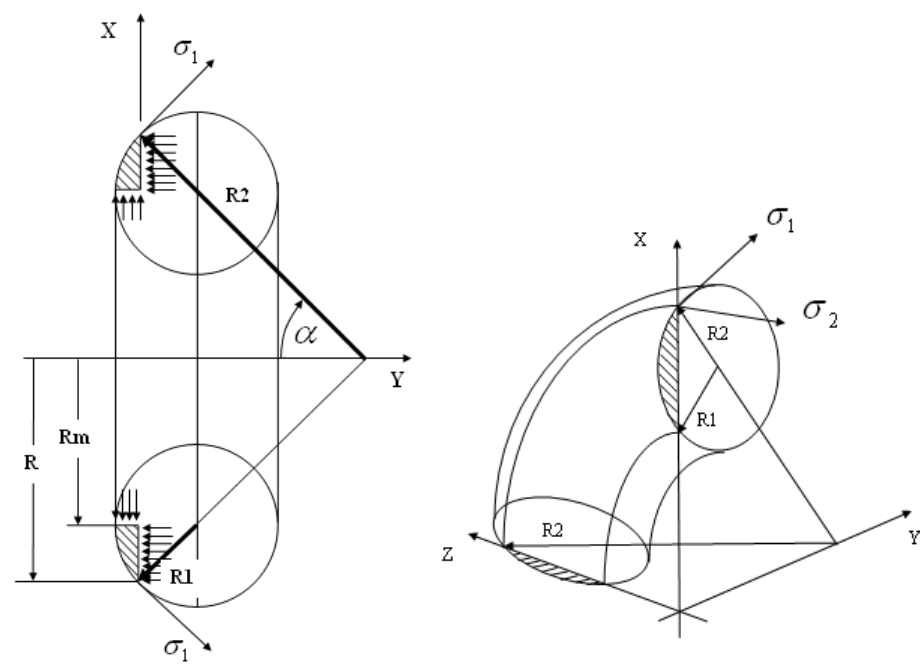

Figure 15: Curvature radii R1, R2 and membrane stress $\sigma_{1}, \sigma_{2}$ in a torus structure.

In eq. 7, parameter $\mathrm{t}$ indicates the wall thickness of the membrane and $\mathrm{p}$ is the pneumatic pressure. Considering equilibrium in the $\mathrm{y}$ - direction leads to the radial stress $\sigma_{1}$.

$2 \pi \cdot R \cdot t \cdot \sigma_{1} \sin \alpha=p \cdot \pi \cdot\left(R^{2}-R m^{2}\right)$

Using the geometric relation

$\sin \alpha=\frac{R-R m}{R 1}$ 
the radial stress of the sidewall is found equal to

$$
\sigma_{1}=p \cdot \frac{R 1}{2 t} \cdot\left(1+\frac{R m}{R}\right)
$$

The circumferential belt force, Fg, is derived considering equilibrium in the z-direction, as shown in Figure 16. A cylindrical shell element representing the belt rests in equilibrium under the action of sidewall stress, $\sigma_{1}$, circumferential belt force, Fg, and pneumatic pressure, p. This situation is expressed by equation (11):

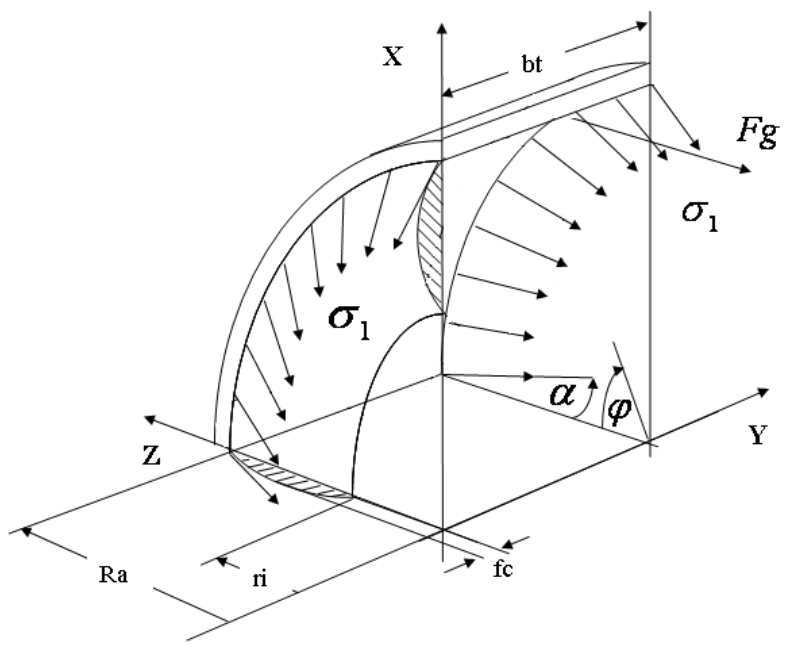

Figure 16: Belt shell structure subjected to sidewall stress $\sigma_{1}$ and belt force Fg.

$F g=p \cdot R a \cdot b t-2 \cdot \sigma_{1} \cos \alpha \cdot R a \cdot t \int_{\varphi=0}^{\pi / 2} \cos \varphi \cdot d \varphi$

Using equation (10) and the geometrical relations (12)

$\begin{array}{rlrl}R m & =\frac{R a+r i}{2} & h c & =R a-r i \\ R 1=\frac{h c^{2}+4 f c^{2}}{8 f c} & \cos \alpha & =\frac{R 1-f c}{R 1}\end{array}$

integration of (11) leads to the circumferential belt force, Fg, as follows:

$F g=p \cdot[R a \cdot b t-(R a+R m) \cdot(R 1-f c)]$

Using equation (10) the sidewall line loads Qsg and Qsr at the belt and rim attachments are

$$
\begin{aligned}
& Q s g=\sigma_{1(R=R a)} \cdot t=p \cdot \frac{R 1}{2} \cdot\left(1+\frac{R m}{R a}\right) \\
& Q s r=\sigma_{1(R=r i)} \cdot t=p \cdot \frac{R 1}{2} \cdot\left(1+\frac{R m}{r i}\right)
\end{aligned}
$$

\subsection{Force response against an edge type radial tyre deformation}


For modeling purposes the DOK manoeuvre is simplified to a drive-over-blade situation whereby the tyre rolls over a blade-type obstacle. The associated tyre force is derived for an idealized edge type line contact to the belt as shown in Figure 17.

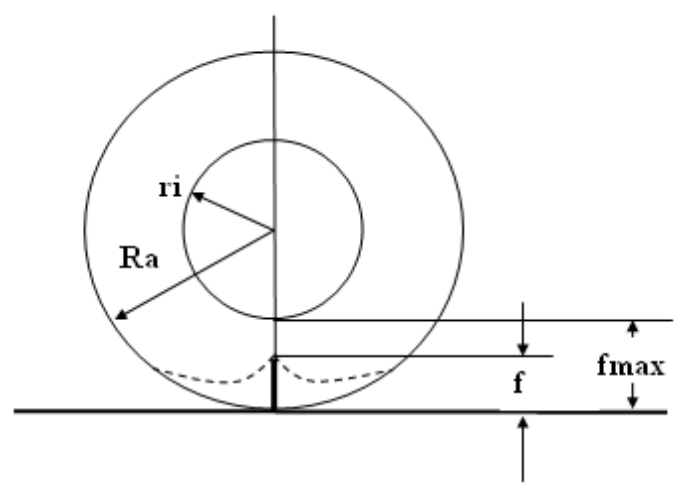

Figure 17: Simplified edge-contact geometry.

As indicated in Figure 16, with increasing radial deflection, $\mathrm{f}$, the projected belt area decreases, as does the associated belt force, Fg. This effect is incorporated by correcting the belt force, Fg, according to equation (16):

$F g_{\text {corr }}=F g \cdot \frac{2 R a-f}{2 R a}$

The horizontal belt force component can be established by equation 16, whereas the vertical force component is unknown in absence of the belt tangential angle. With the assumption of equal belt force magnitudes at the upper and lower belt cross sections, the deformed belt appears to resemble a rope subjected to constant line load distribution per unit length, q, as indicated in Figure 18.

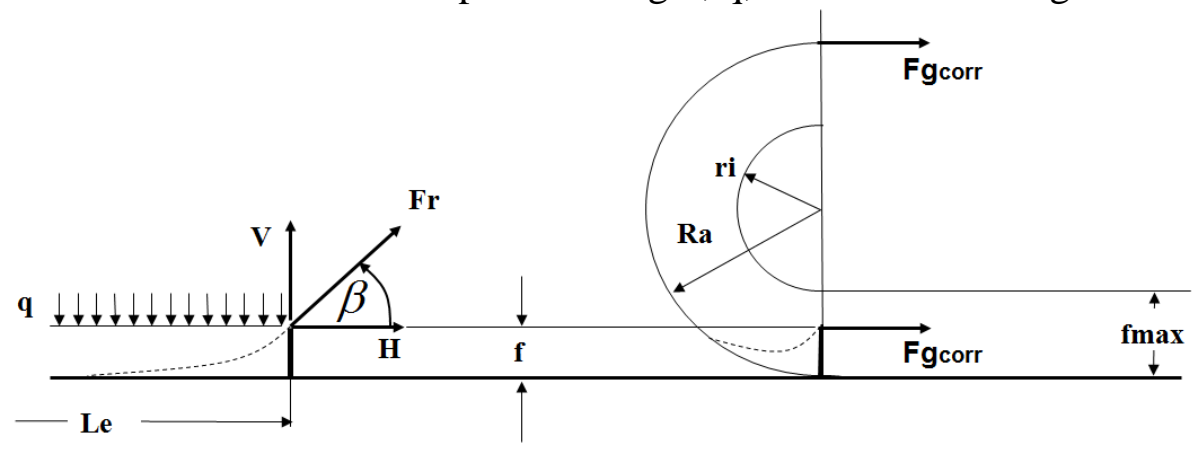

Figure 18: Belt load analogy to rope equation.

The rope indicated by the dashed line is shown for half span width (effective length Le). It is subjected to a horizontal force, $\mathrm{H}$. On the right-hand-side of Figure 18, both upper and lower tyre belt crosssections are subjected to horizontal belt forces, $\mathrm{Fg}_{\text {corr }}$. Assuming an edge contact, the belt is considered to behave similarly to a simply supported rope with horizontal force being $H=F g_{\text {corr }}$. Thus, a vertical component $\mathrm{V}$ can be estimated for the belt based on the rope analogy. Taking into account the rope equations (17), as derived for constant line load q [14]

$$
\begin{gathered}
H=\frac{q \cdot L e^{2}}{8 f_{\max }} \quad \text { and } \quad \tan \beta=4 \cdot \frac{f_{\max }}{L e} \\
\text { with } f_{\max }=R a-r i
\end{gathered}
$$


the horizontal belt force at the tyre-blade contact interface can be derived for the maximum deflection fmax as shown in equation (18).

$$
\begin{aligned}
H & =\frac{q \cdot L e^{2}}{8 f_{\max }} \\
& =p \cdot[R a \cdot b t-(R a+R m) \cdot(R 1-f c)] \cdot \frac{2 R a-f_{\max }}{2 R a} \\
& =F g_{\text {corr }}
\end{aligned}
$$

Rearranging equation 18 and substituting $q=p \cdot b t$ provides an estimation of the effective length, Le, for the extreme case of belt-to-rim contact:

$$
\begin{aligned}
& L e=2 \cdot \sqrt{2 \cdot f_{\max } \cdot\left[R a-\frac{(R a+R m) \cdot(R 1-f c)}{b t}\right] \cdot \frac{2 R a-f_{\max }}{2 R a}} \\
& \text { with } f_{\max }=R a-r i
\end{aligned}
$$

The vertical half belt force component V can then be defined by equation (20) for the maximum deflection as follows:

$V=H \cdot \tan \beta$

Le, strictly speaking, is a function of radial belt deflection $\mathrm{f}$. However, the model agreement to physical measurements can be improved by making the assumption that the effective length Le remains constant over the radial belt deflection range, if Le is derived in the condition of maximum deflection fmax in conjunction with the belt force Fg of the un-deformed tyre. This approach leads to equation (21):

$$
L e=2 \cdot \sqrt{2 \cdot f_{\max } \cdot\left[R a-\frac{(R a+R m) \cdot(R 1-f c)}{b t}\right]}
$$

For arbitrary values of edge deflection, $\mathrm{f}$, the radial edge force Fc can be found after $\tan \beta$ has been linearised within the radial deflection range fmax of the rope (belt), (Figure 18), using equations (17,) (18), (20) and (21).

$$
\begin{aligned}
F C & =2 \cdot V \\
& =2 \cdot F g_{c o r r} \cdot \frac{f}{f_{\max }} \cdot \tan \beta \\
& =2 p \cdot b t \cdot f \cdot \frac{(2 R a-f) \cdot \sqrt{R a-(R a+R m) \cdot(R 1-f c) / b t}}{R a \cdot \sqrt{2 \cdot(R a-r i)}}
\end{aligned}
$$

It is noted, that besides the pneumatic pressure p, all other parameters used in equation (22) can be directly derived by equations (11) from four key dimensions taken from the tyre cross-section shown in Figure 14. 


\subsection{Tyre model validation}

The validity of the analytical tyre model was assessed by comparing the predicted radial force with experimentally measured forces, as well as forces obtained by Finite Element Analysis (FEA). Both physical tests and FEA studies were conducted for three nominal pneumatic pressures (1, 2 and 3 bar) and three deflection levels (20, 40 and $60 \mathrm{~mm}$ ). A comparison between FEA, measured and analytical results is provided in Figure 19. Despite the simplicity of the modeling approach, the radial forces calculated analytically agree well with those predicted by FEA and experiments.

Edge contact Force Fc [N] 205/55-R16

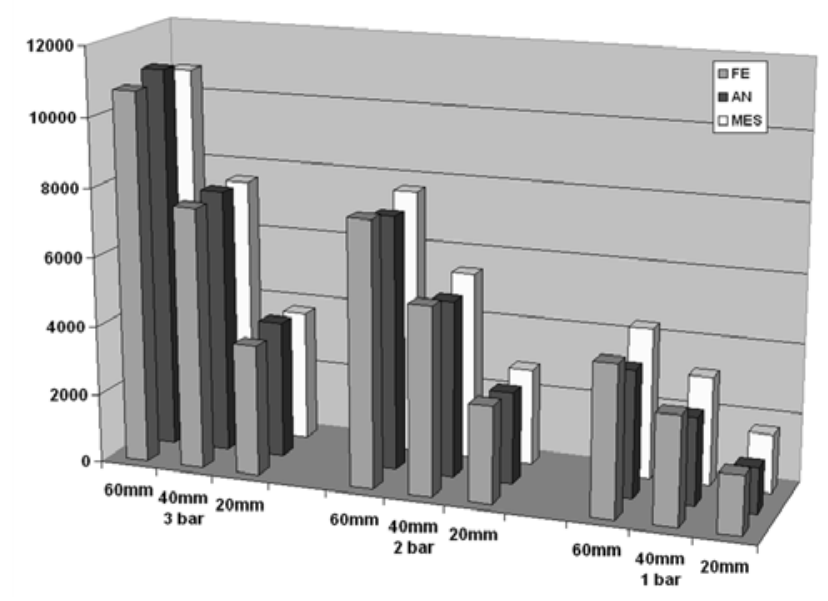

Figure 19: Analytical edge contact force response compared to experimental and FEA results.

\section{TESTING AND SYSTEM LEVEL VALIDATION}

A drive over kerb test as described in the introduction was performed with a CD340 (FORD Galaxy). The test was carried out at two different speeds, namely 25 and $40 \mathrm{kph}$. The vehicle was driven at a free rolling mode over a beam of rectangular cross section of $135 \mathrm{~mm}$ height. The crossing was performed in a direction perpendicular to the beam with the intention of simultaneous impact of both wheels with the bar. The experimental test setup is shown in Figure 20.

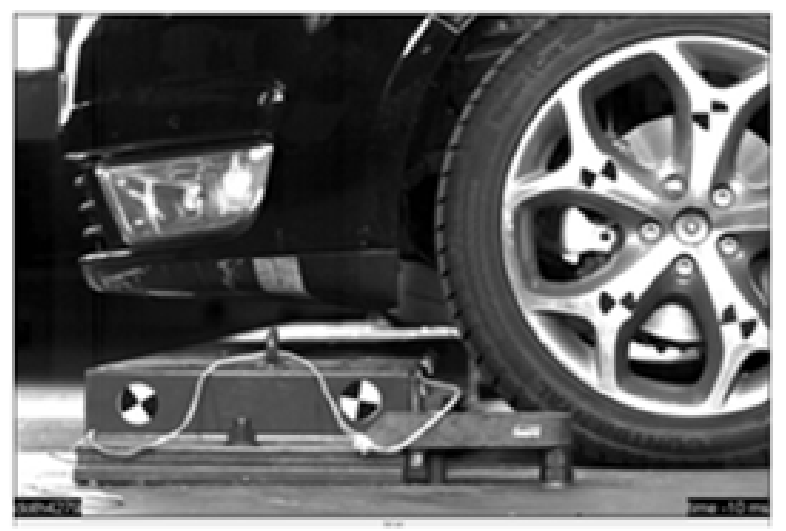

Figure 20: Drive over kerb test set up.

The vehicle was equipped with 235/40-R18 tires operating at a pressure of 2 bar. The vehicle was loaded according to the FORD specification standards for passenger cars.

\subsection{Validation of the full model}

A comparison between the measured (heavy line) and simulated (light line) top mount force timehistories is shown in Figure 21: 

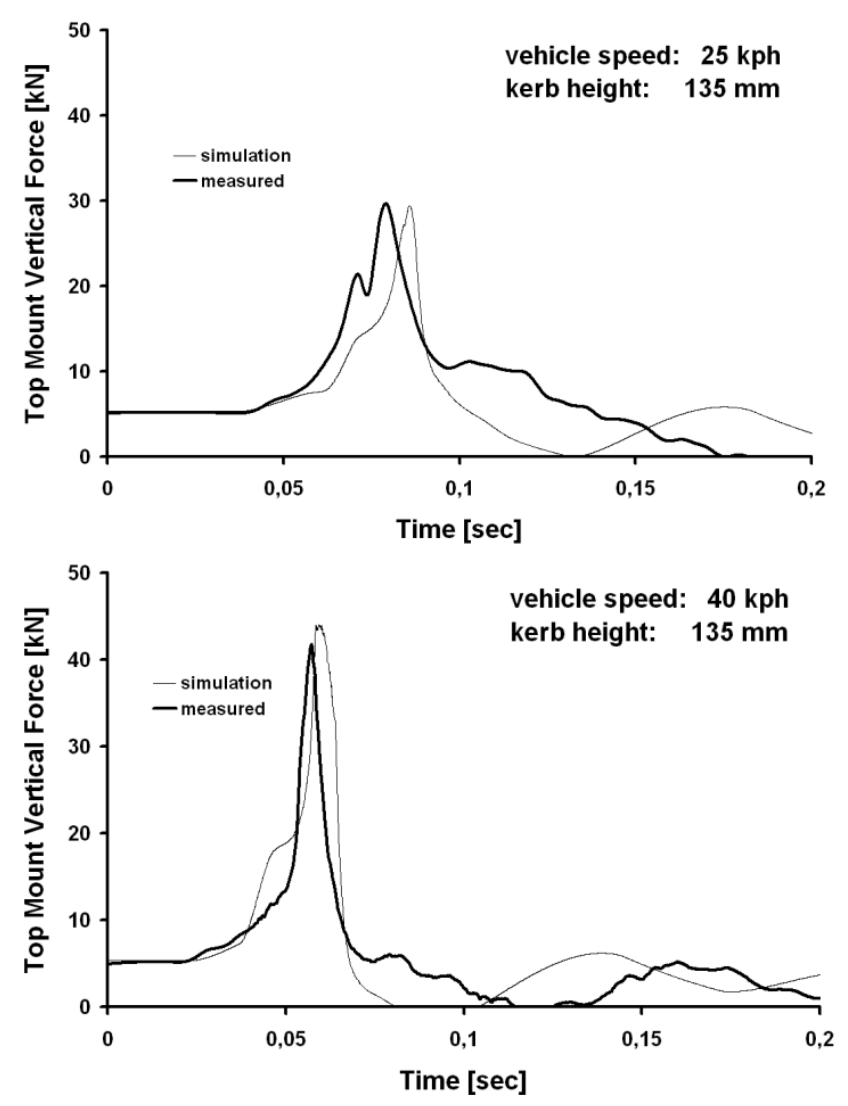

Figure 21: Top mount force and vertical wheel centre deflection over time for drive over kerb test.

The results show very good agreement in terms of force levels. Discrepancies in the actual shape of the experimental and simulation time-histories can be attributed to the simplifications implemented in the model. An additional benefit from the simulation study is that it allows the individual contribution of the spring, damper and bumper to be quantified, as illustrated in Figure 22. The bumper force appears to be the dominant contributor during severe kerb-strike events, as expected. Such is very difficult if not impossible to gain by experimental means alone.

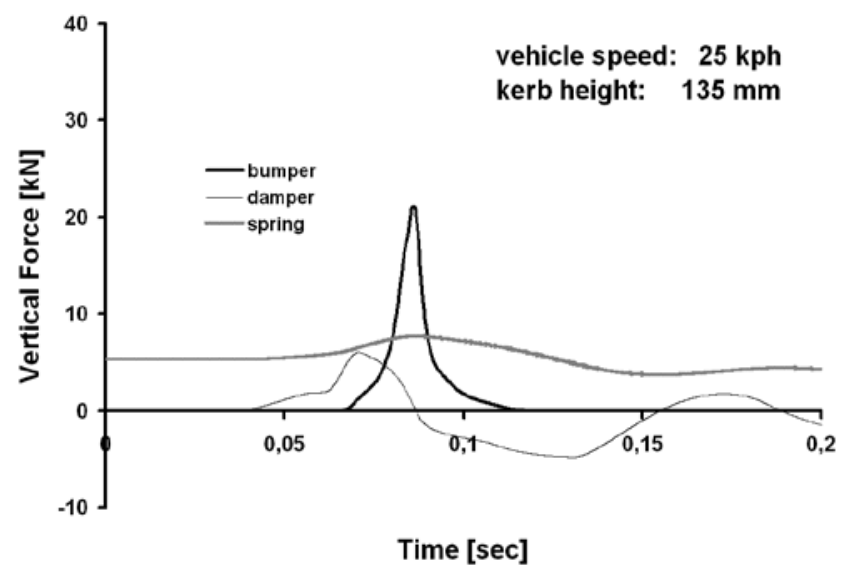




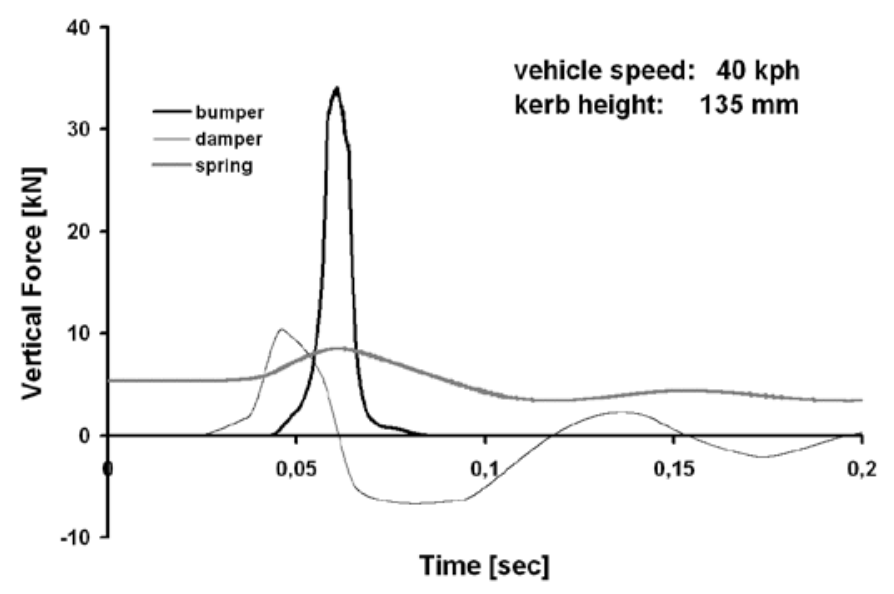

Figure 22: Simulation of spring, damper and bumper force component of top mount force during kerb strike.

\section{CONCLUSION}

A minimal parameter vehicle model has been proposed, specifically tailored to simulate vertical suspension loads during the abusive DOK manoeuvre specified by FORD. Reasonable correlation with experimental data was demonstrated both at component and system levels. Using the proposed simulation tool, parametric studies can be carried out at an early design stage, when only a few design parameters are known. Alternatively, the model can be used later on in the development phase, in order to predict the effect of design changes. In the latter case, calibration of the model based on existing vehicle data would be advisable.

\section{ACKNOWLEDGEMENTS}

This research project has been supported by FORD MOTOR COMPANY. This support is gratefully acknowledged.

\section{REFERENCES}

1 Campbell, C., Automotive suspensions. Chapman Hall, London, 1981.

2 Rahnejat, H., Multi-body dynamics: Vehicles, Machines and Mechanisms. PEP/SAE Joint Publishers, Bury St Edmunds (UK), Warrendale, PA, USA, 1998.

3 Rai, N. s:, Solomon, A. R., Computer Simulation of Suspension Abuse Tests using ADAMS, International Congress \& Exposition, Detroit, Michigan, SAE 820079, 1982.

4 Yoon, J., Kye, K., Suspension Abuse Test Simulation using model stress recovery, SAE 2004-010766, 2004.

5 Austrell, P. E., Wirje, A., A viscoelastic bump stop model for simulations based on impact test data, fifth European conference on Constitutive Models for Rubber (ECCMR), France, 2007. 
6 Jöckel. M., Bruder, F., Franz, T., Charakterisierung und Modellierung des dynamischen Übertragungsverhaltens von Elastomerlagern für die Lastdatenberechnung, Fraunhofer Institut, LBF, Darmstadt, 2008.

7 Gipser, M., ADAMS/Ftire- A Tire Model for Ride \& Durability simulations. International ADAMS User's Conference, Tokyo, 2000.

8 Gallrein, A., Bäcker, M., A tire model for very large tire deformations and its application in very severe events, SAE 2010-10M-0059, 2010.

9 Haga, H., Simulating very large Tire Deformations with CDtire, SAE 2009-01-0577, 2009.

10 Matschinsky, W., Road vehicle suspensions. PEP, Bury St Edmunds, 2000.

11 Willumeit, H. P., Modelle und Modellierungsverfahren in der Fahrzeugdynamik, Teubner Verlag Stuttgart-Leipzig, 1998.

12 Eckstein, L., Vertikal- und Querdynamik von Kraftfahrzeugen, Institut für Kraftfahrzeuge Aachen, Schriftenreihe Automobiltechnik, 2. Auflage, 2011.

13 Timoshenko, S., Strength of materials, part II, advanced problems, second edition, Van Nostrand Comp., 1962.

14 Beer, G., Baustatik 2, Skript zur Vorlesung, Institut für Baustatik TU Graz, S-2-03/2004. 